\title{
Mammogram Order Completion Rates Among Women with Diabetes
}

\author{
Mibir Patel, Matthew Malak, Justin Swanson, MPH, Jennifer Costa, MD, \\ Kea Turner, PhD, MPH, MA, and Karim Hanna, MD
}

Introduction: Breast cancer is one of the leading causes of death among women residing in the United States. Early detection through mammogram screening can decrease the morbidity and mortality associated with the disease. For women with diabetes, however, incidence and mortality rates of breast cancer are increased.

Methods: This was a retrospective examination, identifying orders and completion of mammogram among patients with and without diabetes, 2015 to 2019, through the electronic health record. Diabetes and other factors were identified as possible predictors of completion.

Results: Of the 16,688 patients who were included in this study, only $54.5 \%$ successfully completed mammography orders prescribed by their primary care physician. The 40 to 49 age group had the highest order completion rate at $\mathbf{5 7 . 6 \%}$. The prevalence of diabetes in this sample was $\mathbf{2 1 . 3 \%}$. Overall, $\mathbf{5 1 . 6 \%}$ of patients with diabetes completed mammogram orders compared with $\mathbf{5 5 . 3 \%}$ of patients without diabetes.

Discussion: Patients with diabetes were significantly less likely to complete mammogram screenings (51.6\%) compared with patients without diabetes (55.3\%), despite there being an increased incidence of breast cancer among patients with diabetes. Factors such as the lack of access to treatment centers, affordability, patient education, among others may have contributed to low completion rates. ( $\mathrm{J}$ Am Board Fam Med 2022;35:158-162.)

Keywords: Access to Health Care, Breast Cancer, Diabetes, Family Medicine, Health Care Disparities, Mammography, Outcomes Research, Patient Compliance, Primary Health Care, Screening

\section{Introduction}

Breast cancer is one of the top causes of morbidity and mortality worldwide. According to the World Health Organization, an estimated 2.3 million cases of breast cancer were diagnosed in 2020, and it was the leading cause of cancer-related death for women. ${ }^{1}$ Incidence is even higher in the United States, where 1 in every 8 women is diagnosed with

This article was externally peer reviewed.

Submitted 7 June 2021; revised 20 August 2021; accepted 30 August 2021.

From University of South Florida, Honors College, Tampa, FL (MP, MM); University of South Florida, College of Public Health, Tampa, FL (JS); University of South Florida, Morsani College of Medicine, Department of Family Medicine, Tampa, FL (JC, KH); Moffit Cancer Center, Department of Health Outcomes and Behavior, Tampa, FL (KT).

Funding: This article was not funded by any organization, community, or group.

Conflict of interest: None.

Corresponding author: Karim Hanna, MD, USF Health Morsani College of Medicine, 12901 Bruce B Downs Blvd, Tampa, FL 33612 (E-mail: khanna@usf.edu). some form of invasive breast cancer at some point during their lifetime. ${ }^{2}$ The American Cancer Society has also reported that 1 in 39 American women will die from breast cancer each year. Early detection of breast cancer through regular mammographic screening has been shown to reduce the mortality rate of breast cancer by upward of $20 \%$ among women ages 50 to $74 .^{3,4}$ Therefore, increasing adherence to screening recommendations is an urgent priority. ${ }^{5}$

Individuals with diabetes may be at greater risk of developing breast cancer and may be less likely to adhere to screening guidelines. Studies have found that individuals with diabetes have a $20 \%$ greater risk of developing breast cancer. ${ }^{6,7}$ One of the major reasons behind this is due to the fact that patients with chronic illnesses, such as diabetes, are less likely to engage in routine mammogram screenings. ${ }^{8}$ It may be that managing a complex, chronic illness such as diabetes takes time and resources, which may interfere with preventive 
care. ${ }^{9}$ Factors such as limited health care access, age, socioeconomic status, and race, among others, may also hinder patients with diabetes from completing mammogram screenings. From a pathologic perspective, patients with diabetes have a decreased level of estrogen, which is caused by insulin resistance, leading to a slightly increased risk of developing breast cancer. ${ }^{6}$ Many of the prior studies examining breast cancer screening among women with diabetes are more than a decade old, suffer from small sample sizes, or were conducted outside of the United States, limiting generalizability. ${ }^{8,9}$ Larger and more recent studies are needed to examine whether screening disparities exist for women with diabetes.

To address this gap, the primary objective of this study is to investigate the completion of mammogram orders placed in the primary care setting of US patients with and without diabetes. We seek to identify demographic factors associated with order completion. This data will serve as an epidemiologic basis for understanding the association between diabetes and breast cancer and for future intervention to ultimately help increase completion of screening orders and improve prognosis through early detection.

\section{Methods}

This study includes a retrospective examination of screening mammogram orders through the Epic electronic health record system at the University of South Florida (USF). Mammogram completion was evaluated for adult patients (who generally have access to insurance) established in primary care through the departments of family medicine and internal medicine. Orders placed between August 2015 and August 2019 were examined, and at least 12 months were allowed for follow-up of ordered tests. Orders given to women aged 40 to 49 and 50 to 75 years were included for analysis based on the recommendations for screening of the US Preventative Services Task Force (Grade C and Grade B recommendations, respectively). ${ }^{4}$

The primary relationship of interest was that between diabetes and the completion of mammogram orders. Patients were divided into groups with and without diabetes by ICD-10 codes on patient diagnosis lists. Sociodemographic factors, including age, race/ethnicity, and marital status, were included as covariates. The associations between predictors and mammogram completion are presented as percentages and odds ratios (ORs). Adjusted odds ratios (aORs) and associated 95\% CIs were generated using multivariable logistic regression. Missing data were imputed using multiple imputation by chained equations. ${ }^{10}$ All analyses were executed with $\mathrm{R}$ statistical software version 4.0.5. ${ }^{11}$ Since the study used deidentified datasets for process improvement, it did not represent human subjects research and was exempted from institutional review board approval by USF.

\section{Results}

During the study period, 16,668 patients were given mammogram orders. Of these patients, 3,545 $(21.3 \%)$ had diabetes at the time of the order. Patients with diabetes were older (median, 62; interquartile range [IQR], 55 to 68 ) than patients without diabetes (median, 57; IQR, 49 to 65) and less likely to be married (54.6\% vs $60.9 \%$ ) (Table 1). The sample of patients with diabetes had a smaller percentage of non-Hispanic Whites (45.5\% vs $69.5 \%$ ) and a higher percentage of non-Hispanic Blacks (42.1\% vs $15.5 \%)$.

Overall, $54.5 \%$ of study patients fulfilled their mammogram orders (Table 2). Order completion decreased as age group increased from $57.7 \%$ in the 40 to 49 age group to $52.8 \%$ in the 70 to 74 age group. However, this trend was not statistically significant in the multivariable model (Table 2). White patients recorded the lowest completion rate; both Black (aOR, 1.13; 95\% CI, 1.03-1.23) and Asian (aOR, 1.27; 95\% CI, 1.08-1.50) race were associated with increased odds of order completion. Overall, patients with diabetes experienced a lower likelihood of order completion $(51.6 \%$ vs $55.3 \%)$. After controlling for other factors, patients with diabetes had a decreased odds of order completion (aOR, 0.90; 95\% CI, 0.83-0.98).

\section{Discussion}

Of the 16,668 patients that were included in this study, $54.5 \%$ successfully completed mammography orders prescribed by their primary care physician. Overall, our study found that women with diabetes were less likely $(51.6 \%)$ to complete their mammogram compared with women without diabetes $(55.3 \%)$, consistent with prior studies. 8,9 These findings suggest that interventions may be 
needed to improve mammography screening among women with diabetes, who are at elevated risk for breast cancer.,

The results also indicate that patient race/ethnicity were associated with mammogram completion rates. Healthy People 2020 data suggest that Black women are less likely to undergo cancer mammography screening compared with White women; however, our study did not observe this disparity in screening. ${ }^{12,13}$ It is important to note that Black women are more likely to die from breast cancer than are White women in the United States. ${ }^{14}$ Black women in this study may have generally been insured or had better access to testing facilities, which may have increased the overall compliance in screening. It is also possible that Black women are more likely to complete their mammogram orders but less likely to receive orders in the first place. The 40 to 49 age group had the highest completion rate at $57.6 \%$. These results are different from what previous studies have reported about women 55 and older being more adherent to mammogram screening. ${ }^{15}$

We identified a discrepancy between mammogram orders issued and completion of these orders. Patients with diabetes were less likely to complete mammograms than patients without, with the higher prevalence of breast cancer and mortality among the aforementioned group. This study was conducted among a group of patients who generally have access to insurance, which could be related to the discrepancy. Elsewhere, socioeconomic factors could have had an impact on mammogram completion rates, such as with affordability or transportation to imaging center(s), and these may have been barriers as seen in previous studies. ${ }^{9}$ Patients inadequately educated on the importance of the screening could have also resulted in lower completion percentages. Current literature has noted that certain interventions may enhance screening order completion: reminder-based interventions, culturally tailored interventions, and multifaceted interventions. ${ }^{16}$ These interventions do increase compliance to screening, especially for White patients of higher socioeconomic status. ${ }^{16}$ For patients within the lower income and underinsured group, provider-targeted interventions were found to increase compliance over patient-targeted interventions; physicians that were given reminders/prompts in the electronic health record would always talk to their patients about getting screening. ${ }^{16}$

The study was limited to mammograms that were ordered and completed within the USF Health/Tampa General Hospital System. The study does not consider orders that were completed at other institutions or ordered at other clinics; the completion rates may have been higher than

Table 1. Demographics of Patients Referred for Mammogram in 2015-2019

\begin{tabular}{lccc}
\hline Characteristic & $\begin{array}{c}\text { Overall } \\
\mathrm{n}(\%)\end{array}$ & $\begin{array}{c}\text { Diabetes } \\
\mathrm{n}(\%)\end{array}$ & $\begin{array}{c}\text { No Diabetes } \\
\mathrm{n}(\%)\end{array}$ \\
\hline Total & 16,668 & 3,545 & 13,123 \\
Age & & & $52(55$ to 68$)$ \\
Median, IQR & $58(50$ to 66$)$ & $473(13.3 \%)$ & $57(49$ to 65$)$ \\
40 to 49 & $3846(23.1 \%)$ & $948(26.7 \%)$ & $3373(25.7 \%)$ \\
50 to 59 & $5136(30.8 \%)$ & $1462(41.2 \%)$ & $4188(31.9 \%)$ \\
60 to 69 & $5351(32.1 \%)$ & $662(18.7 \%)$ & $3889(29.6 \%)$ \\
70 to 74 & $2335(14.0 \%)$ & $1938(57.3 \%)$ & $1673(12.7 \%)$ \\
Race/ethnicity & & $913(27.0 \%)$ & $8039(69.5 \%)$ \\
White & $9977(66.7 \%)$ & $120(3.6 \%)$ & $1794(15.5 \%)$ \\
Black & $2707(18.1 \%)$ & $14(0.4 \%)$ & $540(4.7 \%)$ \\
Asian & $660(4.4 \%)$ & $393(11.6 \%)$ & $42(0.4 \%)$ \\
American Indian & $56(0.4 \%)$ & & $1158(10.0 \%)$ \\
Hispanic, all races & $1551(10.4 \%)$ & $1888(54.6 \%)$ & $7052(60.9 \%)$ \\
Marriage status & & $1573(45.4 \%)$ & $4524(39.1 \%)$ \\
Married & $8940(59.5 \%)$ & $6097(40.5 \%)$ & \\
Single & & & \\
\hline
\end{tabular}

Abbreviation: IQR, interquartile range. 
Table 2. Association Between Study Variables and Mammogram Order Completion Among Patients in 2015-2019

\begin{tabular}{lccc}
\hline & \% Completed & Crude OR $(95 \% \mathrm{CI})$ & Adj OR (95\% CI) \\
\hline Covariate & & & \\
Total & $54.5 \%$ & & \\
Age & & & \\
$\quad 40$ to 49 & $57.6 \%$ & Reference & Reference \\
50 to 59 & $54.0 \%$ & $0.86(0.79-0.94)$ & $0.95(0.86-1.04)$ \\
60 to 69 & $53.4 \%$ & $0.84(0.78-0.92)$ & $0.97(0.88-1.06)$ \\
70 to 74 & $52.8 \%$ & $0.82(0.74-0.91)$ & $0.96(0.86-1.08)$ \\
Race/ethnicity & & & Reference \\
White & $52.6 \%$ & Reference & $1.13(1.03-1.23)$ \\
Black & $54.7 \%$ & $1.09(1.00$ to 1.19$)$ & $1.27(1.08-1.50)$ \\
Asian & $58.9 \%$ & $1.30(1.11-1.52)$ & $0.99(0.57-1.71)$ \\
American Indian & $55.4 \%$ & $1.12(0.66-1.91)$ & $1.04(0.93-1.16)$ \\
Hispanic, all races & $53.2 \%$ & $1.03(0.92-1.14)$ & 0.129 \\
Marriage status & & & 0.165 \\
Married & $53.8 \%$ & Reference & 0.021 \\
Single & $52.5 \%$ & $0.95(0.89-1.01)$ & $0.95(0.89-1.02)$ \\
Diabetes & & & Reference \\
No & $55.3 \%$ & $0.86(0.80-0.93)$ & $0.90(0.83-0.98)$ \\
Yes & $51.6 \%$ & & 0.125 \\
\hline
\end{tabular}

Abbreviations: OR, odds ratio; CI, confidence interval.

reported if patients completed them externally, as is generally the case. We also note availability of free mammogram services apart from the USF system possibly impacting internal completion rates. However, we were unable to account for sociodemographic differences associated with screening disparities. Another limitation to consider is that patients with undiagnosed diabetes may have been included in the patient pool, underestimating the rate of diabetes. ${ }^{17}$ Future studies should explore whether sociodemographic differences (AlC level) help to explain observed screening disparities among women with and without diabetes.

\section{Conclusion}

Our study found that mammography screening is lower among women with diabetes, who are at disproportionate risk for breast cancer. Research is needed to identify strategies for promoting screening among women with diabetes. We believe that primary care providers should address this issue by encouraging process improvement initiatives to increase accessibility of cost-cognizant testing, educating patients, and addressing other self-identified barriers to increase mammogram completion.
To see this article online, please go to: http://jabfm.org/content/ 35/1/158.full.

\section{References}

1. Wild CP, Weiderpass E, Stewart BW [Internet]. World cancer report: cancer research for cancer prevention. International Agency for Research on Cancer; 2020 [cited 2021 May 27]. Available from: http://publications.iarc.fr/586.

2. Breast Cancer Facts \& Figures 2019-2020 [Internet]. American Cancer Society; 2019 [cited 2021 May 27]. Available from: https://www.cancer. org/content/dam/cancer-org/research/cancer-factsand-statistics/breast-cancer-facts-and-figures/breastcancer-facts-and-figures-2019-2020.pdf.

3. De Vine CB [Internet]. Mammography. Salem Press Encyclopedia of Health; 2019. [cited 2021 May 27]. Available from: https://search.ebscohost. com/login.aspx?direct=true $\& \mathrm{db}=\mathrm{ers} \& \mathrm{AN}=94462243$ \& site $=$ eds-live.

4. Siu AL, U.S. Preventive Services Task Force. Screening for breast cancer: U.S. Preventive Services Task Force recommendation statement. Ann Intern Med 2016;164:279-96.

5. van Schoor G, Moss SM, Otten JD, et al. Increasingly strong reduction in breast cancer mortality due to screening. Br J Cancer 2011;104: 910-4.

6. Eketunde AO. Diabetes as a risk factor for breast cancer. Cureus 2020;12:e8010. 
7. Lega IC, Austin PC, Fischer HD, et al. The impact of diabetes on breast cancer treatments and outcomes: a population-based study. Diabetes Care 2018;41:755-61.

8. Lipscombe LL, Hux JE, Booth GL. Reduced screening mammography among women with diabetes. Arch Intern Med 2005;165:2090-5.

9. Habeeb SY, Fung K, Fischer HD, Austin PC, Paszat L, Lipscombe LL. Time to follow-up of an abnormal mammogram in women with diabetes: a population-based study. Cancer Med 2016;5: 3292-9.

10. van Buuren S, Groothuis-Oudshoorn K. MICE: multivariate imputation by chained equations in $\mathrm{R}$. J Stat Softw 2011;45.

11. R Foundation [Internet]. The R project for statistical computing; 2021. Available from: https://www. R-project.org/.

12. Rauscher GH, Allgood KL, Whitman S, Conant E. Disparities in screening mammography services by race/ethnicity and health insurance. J Womens Health (Larchmt) 2012;21:154-60.
13. HP2020 Disparities Summary chart [Internet]. Healthy People 2020 website; 2021 [cited 2021 May 27]. Available from: https://www.healthypeople.gov/ 2020/data/disparities/summary/Chart/4055/3.

14. Dobson R. Black women in the US have lower survival rates from breast cancer than white women. BMJ 2008;337(7660):a586.

15. Gathirua-Mwangi W, Cohee A, Tarver WL, et al. Factors associated with adherence to mammography screening among insured women differ by income levels. Womens Health Issues 2018;28: 462-9.

16. Masi CM, Blackman DJ, Peek ME. Interventions to enhance breast cancer screening, diagnosis, and treatment among racial and ethnic minority women. Medical Care Research and Review 2007;64:195S-242S.

17. Prevalence of both diagnosed and undiagnosed diabetes [Internet]. Centers for Disease Control and Prevention; 2020 [cited 2021 June 2]. Available from: https://www.cdc.gov/diabetes/data/statisticsreport/diagnosed-undiagnosed-diabetes.html. 